\title{
Loneliness and Negative Lite Events as Predictors of Hopelessness and Suicidal Behaviors in Hispanics: Evidence for a Diathesis-Stress Model
}

\author{
Edward C. Chang \\ University of Michigan
}

\author{
Lawrence J. Sanna \\ University of North Carolina at Chapel Hill
}

\section{Jameson K. Hirsch}

East Tennessee State University

\section{Elizabeth L. Jeglic \\ John Jay College of Criminal Justice}

In the present study, we examined loneliness and negative life events as predictors of suicide risk (viz., hopelessness and suicidal behaviors) in a sample of 160 Hispanic adults. Consistent with expectations, we found loneliness and negative life events to be positively associated with both hopelessness and suicidal behaviors. In addition, results of conducting hierarchical regression analyses indicated that loneliness accounted for significant amounts of variance in both measures of suicide risk, ranging from $24 \%$ to $29 \%$ of the variance. The inclusion of negative life events as a predictor was found to account for additional unique variance in hopelessness (3\%), but not in suicidal behaviors, beyond what was predicted by loneliness. Finally, consistent with a diathesis-stress model, the Loneliness $\times$ Negative Life Events interaction was found to account for an additional $3 \%$ of the variance in both suicide risk measures. Implications of the present

Edward C. Chang thanks Chang Suk-Choon and Tae Myung-Sook for their encouragement and support throughout this project.

Correspondence concerning this article should be addressed to: Edward C. Chang, Department of Psychology, University of Michigan, 530 Church Street, Ann Arbor, Michigan 48109; e-mail: changec@umich.edu 
findings for future research on suicide risk in Hispanics are discussed. (c) 2010 Wiley Periodicals, Inc. J Clin Psychol 66:1242-1253, 2010.

Keywords: Hispanics; loneliness; life events suicide risk

Hispanics represent one of the fastest growing minority groups in the United States. According to a recent U.S. Census report (U.S. Census Bureau, 2006), Hispanics currently make up the nation's largest minority group and are expected to triple in size over the next four decades. Current projections indicate that by $2050,30 \%$ (132.8 million) of the U.S. population will be Hispanic (U.S. Census Bureau, 2008). Yet, much of the research that examines psychological risk factors in adults continues to be based largely on Whites or European Americans than on other ethnic or racial groups (Graham, 1992). Indeed, although the prediction of suicide risk (e.g., hopelessness, suicide ideation, suicide attempts) has been a focus of hundreds of studies looking at adjustment in adults (e.g., college students; Hass, Hendin, \& Mann, 2003; Westefeld et al., 2006) and comparative findings have indicated that Hispanics may be at greater risk of suicide compared with other racial/ethnic minority groups (Canino \& Roberts, 2001; Olvera, 2001), there have been relatively fewer studies that examined suicide risk in Hispanics (e.g., Del Pilar, 2009; DuartéVélez \& Bernal, 2007; Peña et al., 2008). Accordingly, in the present study, we propose and test a prediction model that involves loneliness and negative life events as predictors of suicide risk in Hispanics.

\section{Loneliness and Negative Life Events: A Proposal for a Model of Suicide Risk in Hispanics}

Loneliness, often due to poor interpersonal relationships, is defined by feelings and thoughts of being isolated and disconnected from others (Russell, Peplau, \& Cutrona, 1980). Thus, one may experience loneliness even as a member of a large group. There are at least three compelling reasons for considering loneliness in a model of suicide risk in adults, and perhaps especially in Hispanic adults. First, loneliness has been linked to a variety of physical and psychological difficulties including depression, anxiety, stress, and physical problems (see Heinrich \& Gullone, 2006, for a review of the relevant literature). Not surprisingly, findings from studies have shown that greater loneliness is associated with greater suicide risk in adults (e.g., Conroy \& Smith, 1983; Stravynski \& Boyer, 2001). Second, loneliness has been identified as one of the major reasons why adults attempt suicide. In one notable study, Westefeld and Furr (1987) found that in $47 \%$ of the adults who indicated a history of suicide ideation, loneliness was the most frequently cited cause of suicide ideation. Third, one of the major considerations in the assessment of suicidal behavior is a consideration of the cultural context of Hispanics. In general, Hispanics are believed to support and maintain collectivistic values (Triandis, Bontempo, Villareal, Asai, \& Lucca, 1988). These values are, for example, rooted in revered notions associated with "mi familia" or "familismo" (e.g., Bernal, CumbaAvilés, \& Saez-Santiago, 2006). Unlike individualistic values held by Whites and European Americans who tend to focus on the development of the independent self (Chang \& Asakawa, 2003; Chang, Asakawa, \& Sanna, 2001), familismo tends to focus on the development of the interdependent self (Duarté-Vélez \& Bernal, 2007). Therefore, the inability to develop and maintain close interpersonal relations with 
others (e.g., family members, friends) should represent an important predictor of suicide risk among Hispanics.

In addition to loneliness, it may be useful to consider the role of negative life events in predicting suicide risk in Hispanics. Of existing studies looking at correlates of suicide risk in Hispanics, many have identified a variety of situational factors (e.g., substance use, sexual abuse, trauma, and acculturative stress; Blume, Resor, Villanueva, \& Braddy, 2009; Peña et al., 2008; Rew, Thomas, Horner, Resnick, \& Beuhring, 2001; Wadsworth \& Kubrin, 2007). Additionally, in a study of Hispanic suicide completers, Queralt (1993) found that based on assessments obtained from family members and school counselors, completers (compared to a nonsuicidal Hispanic control group) experienced greater life stress as indicated by greater school, personality, behavioral, and family problems. These patterns are consistent with findings, obtained in the general adult population, that demonstrate a reliable positive association between measures of negative life events and suicide risk (e.g., Joiner \& Rudd, 2000; Lewinsohn, Rohde, \& Seeley, 1996). Accordingly, one might expect that, beyond loneliness, negative life events would uniquely contribute to the prediction of suicide risk in Hispanics.

Finally, beyond these considerations, we believe there is value in looking at a diathesis-stress model, which involves the interaction of loneliness and negative life events in predicting suicide risk in Hispanics. That is, although loneliness and negative life events may represent important and independent explanatory variables linked to suicide, they may also interact in predicting suicide risk. Indeed, findings from past studies have shown that personality or individual differences variables (e.g., perfectionism, social problem solving, and dispositional optimism) often interact with negative life events in predicting a range of negative outcomes and conditions, including suicide risk (e.g., Bonner \& Rich, 1988; Chang \& Rand, 2000; Hirsch, Wolford, LaLonde, Brunk, \& Morris, 2007). Indeed, in a study that involved Asians, a group also known be to collectivistic, Yang and Clum (1994) found that loneliness and negative life events interacted in predicting suicide ideation. To date, however, no study has examined the explanatory power of loneliness and negative life events as predictors of suicide risk in Hispanic adults.

\section{Purpose of the Present Study}

Given these considerations, we examined the role of loneliness and negative life events as predictors of suicide risk, namely, hopelessness and suicidal behaviors, in Hispanics. We had four specific objectives: (a) to examine the relations between loneliness, negative life events, hopelessness, and suicidal behaviors; (b) to determine if loneliness accounts for significant variance in hopelessness and suicidal behaviors; (c) to determine if the inclusion of negative life events would significantly add to the prediction of additional unique variance, beyond what may be accounted for by loneliness, in predicting hopelessness and suicidal behaviors; and (d) to determine if there was a significant Loneliness $\times$ Negative Life Events interaction that would account for further unique variance in predicting hopelessness and suicidal behaviors.

Based on our earlier discussion, loneliness and negative life events were expected to be positively related to both hopelessness and suicidal behaviors in Hispanics. Loneliness in Hispanics was predicted to account for a significant amount of the variance in each of the two suicide risk measures. Relatedly, we expected the inclusion of negative life events to significantly augment the prediction model of 
suicide risk in Hispanics. Finally, consistent with a diathesis-stress model, we hypothesized that there would be a significant Loneliness $\times$ Negative Life Events interaction in predicting suicide risk in Hispanics. Specifically, we predicted the interaction would show that the expected positive association between loneliness and suicide risk would be stronger among Hispanics who experience high compared with low negative life events.

\section{Method}

\section{Participants}

A total of 160 (38 men and 122 women) self-identified Hispanic adults, attending a large public college in the East Coast, participated in the present study. All participants were enrolled in an introduction to psychology course and earned research credit for participating. The ages ranged from 18 to 46 years (mean $[M]=19.78$, standard deviation $[S D]=3.70$ ).

\section{Measures}

Loneliness. Loneliness was assessed by the revised UCLA Loneliness Scale (R-UCLA; Russell et al., 1980). The scale comprise 20 items, half of which is worded positively (e.g., "There are people I feel close to"), while the other is worded negatively (e.g., "I feel isolated from others"). Respondents are asked to rate the statements on the frequency with which they experience these feelings, using a 4-point Likert-type scale, ranging from 1 (never) to 4 (often). Research has shown the UCLA scale to have convergent validity with other measures of loneliness (see Russell, 1996). In the present sample, internal reliability for the R-UCLA was .89. Average interitem correlation was .33. Higher scores on the R-UCLA indicate greater levels of loneliness.

Negative life events. Negative life events were assessed by the Life Events Checklist for College Students (LECCS; Tomoda, 1997). The LECCS is a 46-item self-report measure of various life events encountered by college students (e.g., "Had financial difficulties," "Failed in studies," "Had difficulties with a family member"). Respondents are asked to indicate whether they had experienced each life event over the past year. The LECSS provides scores for the number of positive and negative life events experienced. However, given our interest in negative life events (NEG), we focused only on LECCS-NEG scores. In the present sample, internal reliability for the LECCS-NEG was .73 (low reliability estimates are common for life events measures that tap for a wide range of unrelated events). Average interitem correlation was .12. Higher scores on the LECCS-NEG indicate greater experience of negative life events.

Hopelessness. Hopelessness was assessed by the Beck Hopelessness Scale (BHS; Beck, Weissman, Lester, \& Trexler, 1974). The BHS is a 20-item measure of extreme pessimism or hopelessness. Respondents are asked to indicate either agreement or disagreement to these items that assess negative expectancies for the future (e.g., "My future seems dark to me"). Scores on the BHS have been found to correspond highly with clinical ratings of hopelessness (Beck et al., 1974) and have also been found to predict eventual suicides (Beck, Steer, Kovacs, \& Garrison, 1985). In the present sample, internal reliability (KR-20) for the BHS was .91. Average interitem correlation was .34. Higher scores on the BHS are indicative of greater hopelessness. 
Suicidal behaviors. Suicidal behaviors were assessed by the Suicidal Behaviors Questionnaire-Revised (SBQ-R; Osman et al., 2001). The SBQ-R is a 4-item measure of lifetime suicide ideation and attempt ("Have you ever thought about or attempted to kill yourself?"), frequency of suicide ideation in the last year ("How often have you thought about killing yourself in the past year?"), threat of suicidal behavior ("Have you ever hold someone that you were going to commit suicide, or that you might do it?"), and likelihood of future suicidal behavior ("How likely is it that you will attempt suicide someday?"). Respondents are asked to answer each question on the frequency with which they engaged in the suicidal behavior, using a Likert-type scale. For example, respondents are asked to indicate their frequency of having suicidal ideations, ranging from 1 (never) to 5 (very often). Scores on the SBQ-R have been found to differentiate between suicidal and nonsuicidal adults (Osman et al.). In the present sample, internal reliability for the SBQ-R was .80. Average interitem correlation was .59. Higher scores on the SBQ-R are indicative of greater suicidal behaviors.

\section{Procedure}

A survey battery that included the R-UCLA, LECCS-NEG, BHS, and SBQ-R scales were given to all participants. These scales were randomly ordered. Participants were not made aware of the purpose of the study until after the study was completed. All participants signed consent forms that indicated that all test data would be kept strictly confidential.

\section{Results}

Results of computing correlations, means, standard deviations, and reliability estimates for all study measures are presented in Table 1. As expected, scores on the R-UCLA were found to be significantly and positively associated with BHS and SBQ-R scores in the large effect size range ( $r \mathrm{~s}=.49$ to .54$)$. Similarly, scores on the LECCS-NEG were also found to be significantly and positively associated with BHS and SBQ-R scores in the moderate effect size range $(r \mathrm{~s}=.30$ to .35$)$. It is noteworthy that R-UCLA and LECCS-NEG scores were also found to be significantly and positively associated $(r=.36)$, indicating that greater loneliness involves greater frequency of experiencing negative life events. Finally, as measures of suicide risk, BHS and SBQ-R scores were found to significantly and positively associated

Table 1

Zero-Order Correlations, Means, and Standard Deviations of Study Measures

\begin{tabular}{lcccc}
\hline & R-UCLA & LECCS-NEG & BHS & SBQ-R \\
\hline R-UCLA & - & & & \\
LECCS-NEG & $.36^{* * *}$ & - & & \\
BHS & $.54^{* * *}$ & $.35^{* * *}$ & - & - \\
SBQ-R & $.49^{* * *}$ & $.30^{* * *}$ & $.50^{* * *}$ & 1.79 \\
$M$ & 43.60 & 6.76 & 4.18 & 3.22 \\
$S D$ & 8.90 & 3.67 & 4.83 & \\
\hline
\end{tabular}

Note: UCLA-R = Revised UCLA Loneliness Scale; LECCS-NEG = Life Events Checklist for College Students-Negative Life Events; BHS = Beck Hopelessness Scale; SBQ-R = Suicidal Behavior QuestionnaireRevised; $\mathrm{M}=$ mean; $\mathrm{SD}=$ standard deviation. $N=160$.

$* * * p<.001$. 
$(r=.50)$, but the association was not so high as to indicate redundancy in measurement.

Loneliness and Negative Life Events as Predictors of Hopelessness and Suicidal Behaviors in Hispanics

To examine the predictive utility of loneliness and negative life events in accounting for variance in hopelessness and suicidal behaviors in Hispanics, we conducted a series of hierarchical regression analyses predicting scores on each of the suicide risk measures. For each of these regression equations, UCLA-R scores and LECCSNEG scores were entered as predictors of suicide risk in the first and second Step, respectively. Finally, to test for an interaction model, UCLA-R $\times$ LECCS-NEG scores were entered in the final step as predictors of suicide risk. Results of these analyses for predicting variance in hopelessness and suicidal behaviors are presented in Table 2. To determine if loneliness and negative life events accounted for a small, medium, or large amount of the variance in functioning, we used Cohen's (1977) convention for small $\left(f^{2}=.02\right)$, medium $\left(f^{2}=.15\right)$, and large effects $\left(f^{2}=.35\right)$.

As the table shows, scores on the UCLA-R were found to account for a large $\left(f^{2}=.40\right)$ and significant $29 \%$ of the variance in hopelessness. After partialing out the variance accounted for by loneliness, LECCS-NEG scores were found to account for an additional small $\left(f^{2}=.03\right)$ but significant $3 \%$ of the variance in hopelessness. Furthermore, the interaction term that involves UCLA-R $\times$ LECCS-NEG was found to account for an additional small $\left(f^{2}=.02\right)$ but significant $2 \%$ of the variance in hopelessness. The total prediction model was found to account for a large $\left(f^{2}=.49\right)$ and significant $33 \%$ of the variance in hopelessness in Hispanics, $F(3,156)=26.15, p<.001$.

Similarly, scores on the UCLA-R were also found to account for a large $\left(f^{2}=.32\right)$ and significant $24 \%$ of the variance in suicidal behaviors. After partialing out the variance accounted for by loneliness, LECCS-NEG scores were found to account for an additional small $\left(f^{2}=.02\right)$ but nonsignificant $(p>.05) 2 \%$ of the variance in suicidal behaviors. Furthermore, the interaction term that involves UCLA-R $\times$ LECCS-NEG was found to account for an additional small $\left(f^{2}=.03\right)$ but significant $3 \%$ of the variance in suicidal behaviors. The total prediction model was found to

Table 2

Results of Hierarchical Regression Analyses-Amount of Variance in Hopelessness and Suicidal Behaviors Accounted for by Loneliness, Negative Life Events, and the Loneliness $\times$ Negative Life Events Interaction

\begin{tabular}{llllrr}
\hline Outcome and measure & $\beta$ & $R^{2}$ & $\Delta R^{2}$ & $F$ & $p$ \\
\hline BHS & & & & & \\
$\quad$ Step 1: UCLA-R & $.54^{* * *}$ & .29 & & 62.85 & $<.001$ \\
Step 2: LECCS-NEG & $.19^{* *}$ & .32 & .03 & 6.77 & $<.01$ \\
Step 3: UCLA-R $\times$ LECCS-NEG & $.90^{*}$ & .33 & .02 & 4.75 & $<.05$ \\
SBQ-R & & & & & \\
Step1: UCLA-R & $.49^{* * *}$ & .24 & & 49.93 & $<.001$ \\
Step 2: LECCS-NEG & .14 & .26 & .02 & 3.77 & ns \\
Step 3: UCLA-R $\times$ LECCS-NEG & $1.05^{*}$ & .29 & .03 & 5.99 & $<.05$ \\
\hline
\end{tabular}

Note: UCLA-R = Revised UCLA Loneliness Scale; LECCS-NEG = Life Events Checklist for College Students-Negative Life Events; BHS = Beck Hopelessness Scale; SBQ-R = Suicidal Behavior QuestionnaireRevised; ns = nonsignificant. $N=160$.

${ }^{*} p<.05 ;{ }^{* *} p<.01 ;{ }^{* * *} p<.001$. 


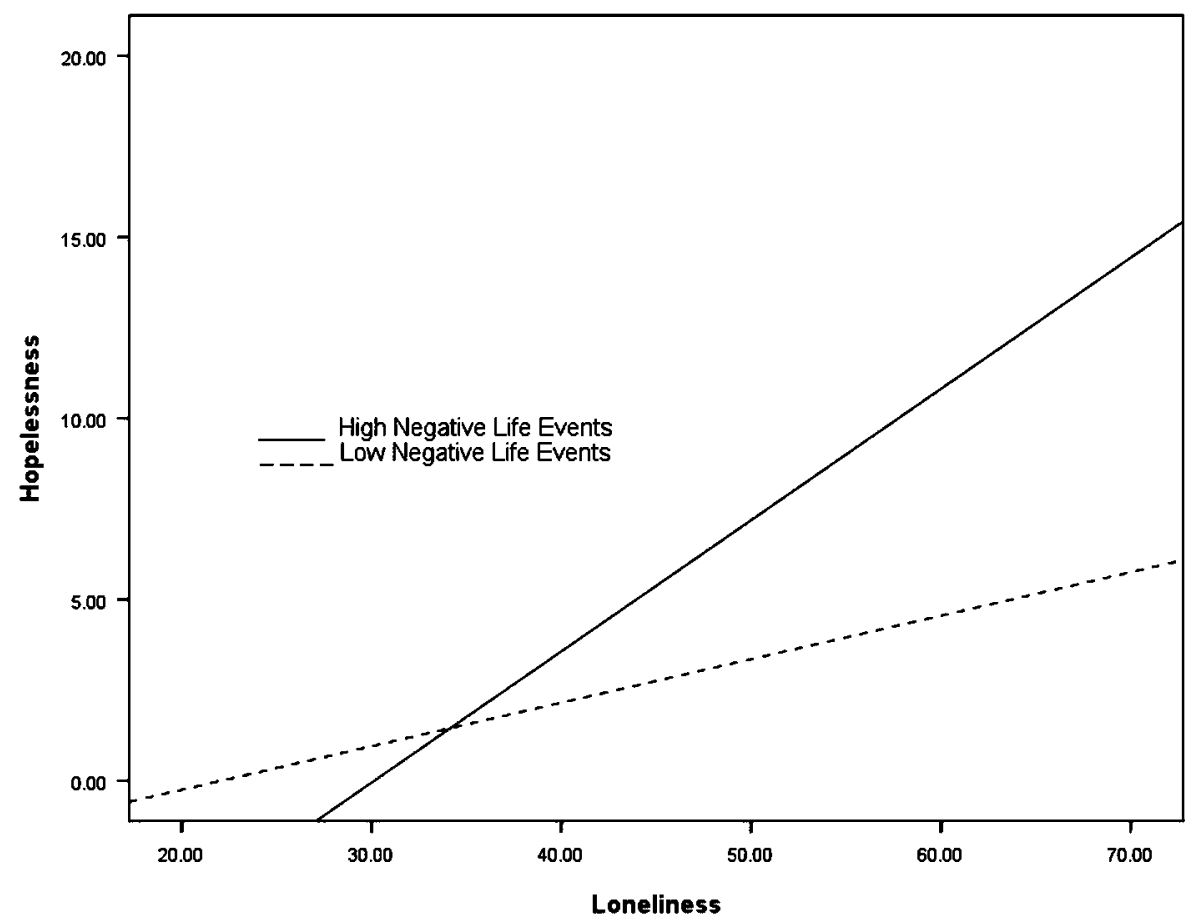

Figure 1. Relationship of loneliness to hopelessness at high and low levels of negative life events $(n=160)$.

account for a large $\left(f^{2}=.41\right)$ and significant $29 \%$ of the variance in suicidal behaviors in Hispanics, $F(3,156)=20.77, p<.001$.

To visually inspect the manner in which loneliness and negative life events interacted with each other in predicting suicide risk, we plotted the regression of loneliness on hopelessness at low and high levels (1 SD below and above the mean, respectively) of negative life events based on our initial regression results. Results of plotting these interactions were consistent with our hypotheses. Specifically, the presence of high compared with low negative life events appeared to increase the positive association present between loneliness and hopelessness (Fig. 1). Similarly, the presence of high compared to low negative life events again appeared to increase the positive association present between loneliness and suicidal behaviors (Fig. 2).

Overall, the results of our regression analyses indicate that loneliness and negative life events do significantly, additively, and interactively contribute to the prediction of hopelessness in Hispanics. Additionally, our results indicate that loneliness significantly and interactively, with negative life events, predict suicidal behaviors in Hispanics.

\section{Discussion}

We conducted the present study to examine a model of suicide risk in Hispanics predicated on loneliness and negative life events. As noted earlier, despite evidence for the explanatory power of loneliness and negative life events in predicting suicide risk in the general adult population, these variables have never been examined in tandem as potential predictors of hopelessness and suicidal behaviors in Hispanics.

Consistent with expectations, loneliness and negative life events were found to be associated with greater hopelessness and suicidal behaviors in Hispanics. 


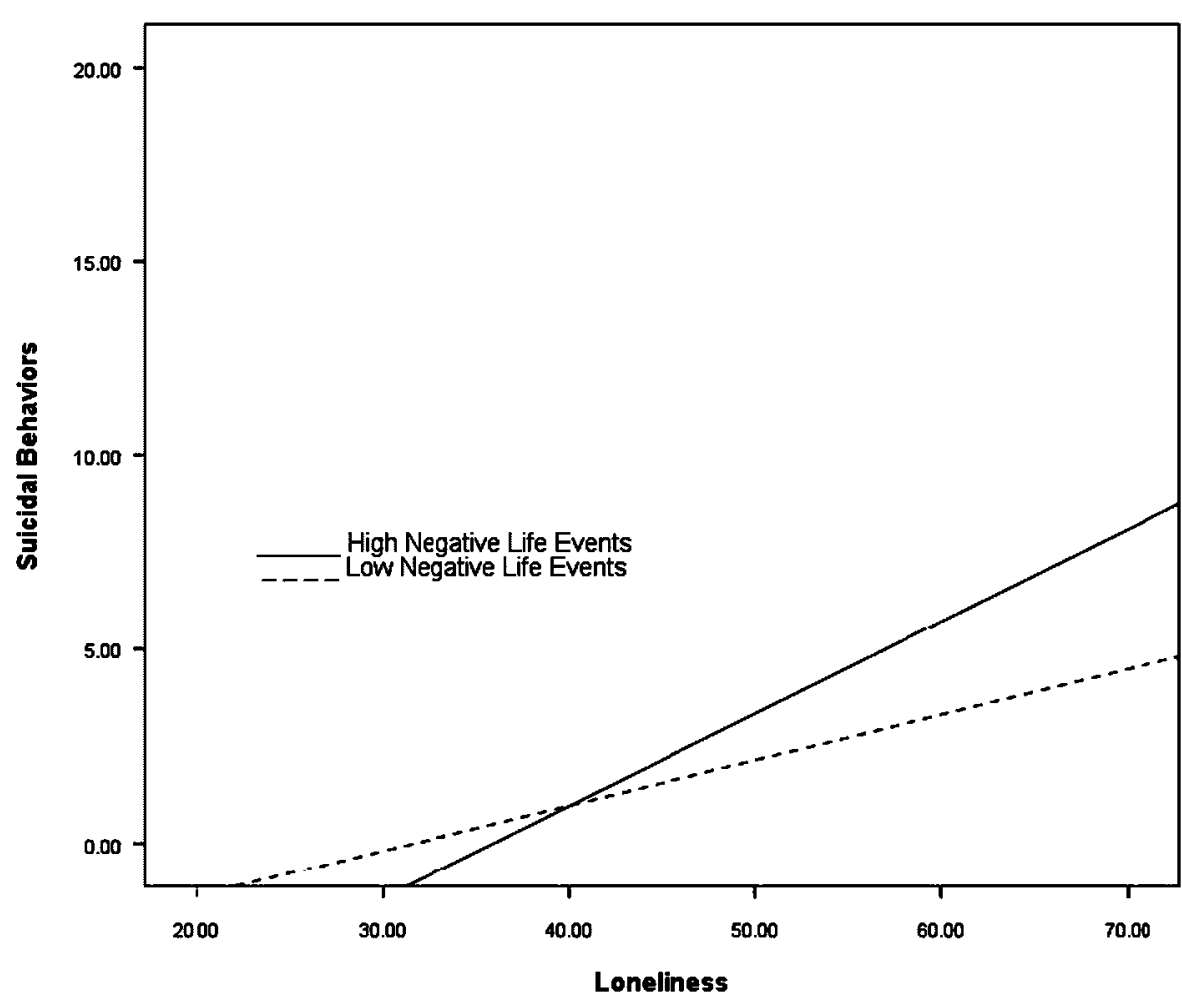

Figure 2. Relationship of loneliness to suicidal behaviors at high and low levels of negative life events $(n=160)$.

Interestingly, associations that involved loneliness with suicide risk were found to be generally stronger (large effect sizes) than those that involved negative life events with suicide risk (medium effect sizes). As noted earlier, the ability to develop and maintain close interpersonal relationships associated with collectivist values like familismo (Bernal et al., 2006) may be particularly important for Hispanics.

\section{Loneliness and Negative Life Events: A Diathesis-Stress Model of Suicide Risk in Hispanics}

In conducting regression analyses, we found that loneliness in Hispanics accounted for a significant amount of the variance in each of the two suicide risk measures, with negative life events adding to the regression model in predicting hopelessness, but not in predicting suicidal behaviors. However, consistent with a diathesis-stress model, the Loneliness $\times$ Negative Life Events interaction term was found to account for a small but significant amount of additional variance in predicting both hopelessness and suicidal behaviors in Hispanics. Thus, our findings point to a need to consider the complex interplay between loneliness and negative life events in conceptualizing predictors of suicide risk in Hispanic adults. For example, as noted earlier, loneliness is believed to typically result from poor or negative interpersonal experiences. It may be that among Hispanics that feel burdened by overly strong collectivist pressures such as excessive familism (familismo excesivo), the experience of negative personal life events for a Hispanic adult may be taken by other family members as a general failure of the individual to meet the social expectations and 
needs of the collective family (Pantin et al., 2003). In turn, these experiences may contribute to not only their greater feelings of loneliness but also the development of greater suicide risk over time.

It is important to note that the present findings are based on a convenience sample, rather than a clinical sample, of Hispanic adults. In other words, one might argue that the present findings speak more about suicide risk potential than it does of actual suicide risk or suicide behaviors. Indeed, although $85 \%$ of the present sample $(n=136)$ indicated experiencing some form of hopelessness, the average BHS score was low $(M=4.83)$, compared with scores found to be predictive of actual suicide attempts in clinical samples $(M \geq 0$; Beck et al., 1985). However, this should not be taken to suggest that there is little importance in assessing and treating subclinical levels of suicidal risk and distress in Hispanic adults. The presence of forme fruste, subclinical or low-level distress, among even "healthy" Hispanics may indicate a situation in which clinically significant dysfunctions may later develop over time. According to Del Pilar (2009), because the presence of forme fruste is often overlooked or untreated in "normal" Hispanic adults, individuals with this condition remain continually frustrated and unable to function to their full potential, placing them at greater risk for more serious problems later on. Accordingly, our findings suggest that mental health efforts to develop early detection programs and interventions that focus on the prevention of suicide in Hispanic adults may benefit from not only integrating a greater appreciation for the role of loneliness and negative life stress but also routine assessments of these important experiences in this population. Nonetheless, it would be important in future studies to examine the present prediction model in a clinical population to determine if important differences emerge.

Beyond these considerations, it is worth noting some limitations of the present study. First, the focus of our study was on Hispanics as a general group. Because Hispanics are represented by diverse subgroups (Guarnaccia et al., 2007), it is important to evaluate the generalizability of the present findings in more specific groups (e.g., Mexican Americans, Cubans, Puerto Ricans, Dominicans). Second, given that loneliness and negative life events have been linked to a range of other important outcomes (e.g., depression, anxiety), it would be valuable in future studies to determine the usefulness of the present model for predicting other outcomes relevant to Hispanics. Third, it may be useful to consider both risk and resilience factors in future research (Gutierrez, 2006). For example, it may be worth examining the potential buffering effects of positive life events on the link between loneliness and suicide risk in Hispanics. Fourth, it would be important in future studies to see how culture-specific variables (e.g., ethnic identity, acculturation) might interact with or add to a general model of loneliness and negative life events in the prediction of suicide risk in Hispanics. Finally, given the cross-sectional nature of the present study, it would be important in future studies to use longitudinal designs that can help clarify the causal associations that involve loneliness and negative life events with suicide risk in Hispanic adults, as well as identify important underlying mechanisms (e.g., dysfunctional problem solving; Chang, 2002; D'Zurilla, Chang, Nottingham, \& Faccini, 1998) that may account for their associations.

\section{Concluding Thoughts}

In summary, we looked at loneliness and negative life events as predictors of suicide risk in Hispanics. We found that loneliness was an important and robust predictor of 
both hopelessness and suicidal behaviors. In addition, we found that the inclusion of negative life events significantly augmented the prediction model directly or interactively, beyond loneliness, in accounting for hopelessness and suicidal behaviors. Moreover, we found support consistent with a diathesis-stress model that involves loneliness and negative life events. By focusing on potential factors linked to suicide risk in Hispanic adults, we hope that our findings will help future researchers to not only develop and test more comprehensive models in future investigations but also culturally useful approaches to prevent and treat suicide risk in this important and burgeoning population.

\section{References}

Beck, A.T., Steer, R.A., Kovacs, M., \& Garrison, B. (1985). Hopelessness and eventual suicide: A 10-year prospective study of patients hospitalized with suicidal ideation. American Journal of Psychiatry, 142, 559-563.

Beck, A.T., Weissman, A., Lester, D., \& Trexler, L. (1974). The measurement of pessimism: The Hopelessness Scale. Journal of Consulting and Clinical Psychology, 42, 861-865.

Bernal, G., Cumba-Avilés, E., \& Saez-Santiago, E. (2006). Cultural and relational process in depressed Latino adolescents. In: S.R.H. Beach, M.Z. Wamboldt, N.J. Kaslow, R.E. Heyman, \& D. Reiss (Eds.), Relational processes and DSM-V: Neuroscience, assessment, prevention and treatment (pp. 221-224). Washington, DC: American Psychiatric Association.

Blume, A.W., Resor, M.R., Villanueva, M.R., \& Braddy, L.D. (2009). Alcohol use and comorbid anxiety, traumatic stress, and hopelessness. Addictive Behaviors, 34, 709-713.

Bonner, R.L., \& Rich, A. (1988). Negative life stress, social problem-solving self-appraisal, and hopelessness: Implications for suicide research. Cognitive Therapy and Research, 12, $547-556$.

Canino, G., \& Roberts, R.E. (2001). Suicidal behavior among Latino youth. Suicide and LifeThreatening Behavior, 31, 122-131.

Chang, E.C. (2002). Pre Predicting suicide ideation in an adolescent population: Examining the role of social problem solving as a moderator and a mediator. Personality and Individual Differences, 32, 1279-1291.

Chang, E.C., \& Asakawa, K. (2003). Cultural variations on optimistic and pessimistic bias for self versus a sibling: Is there evidence for self-enhancement in the West and self-criticism in the East when the referent group is specified? Journal of Personality and Social Psychology, 84, 569-581.

Chang, E.C., Asakawa, K., \& Sanna, L.J. (2001). Cultural variations in optimistic and pessimistic bias: Do Easterners really expect the worst and Westerners really expect the best when predicting future life events? Journal of Personality and Social Psychology, 81, 476-491.

Chang, E.C., \& Rand, K.L. (2000). Perfectionism as a predictor of subsequent adjustment: Evidence for a specific diathesis-stress mechanism among college students. Journal of Counseling Psychology, 47, 129-237.

Cohen, J. (1977). Statistical power analysis for the behavioral sciences (rev. ed.). New York: Academic Press.

Conroy, R.W., \& Smith, K. (1983). Family loss and hospital suicide. Suicide and LifeThreatening Behavior, 13, 179-194.

Del Pilar, J.A. (2009). Mental health and Latino/a college students: A psychological perspective and new findings. Journal of Hispanic Higher Education, 8, 263-281.

Duarté-Vélez, Y.M., \& Bernal, G. (2007). Suicide behavior among latino and latina adolescents: Conceptual and methodological issues. Death Studies, 31, 435-455. 
D'Zurilla, T.J., Chang, E.C., Nottingham IV, E.J., \& Faccini, L. (1998). Social problemsolving deficits and hopelessness, depression, and suicidal risk in college students and psychiatric inpatients. Journal of Clinical Psychology, 54, 1091-1107.

Graham, S. (1992). "Most of the subjects were White and middle class": Trends in published research on African Americans in selected APA journals, 1970-1989. American Psychologist, 47, 629-639.

Guarnaccia, P.J., Pincay, I.M., Alegría, M., Shrout, P.E., Lewis-Fernández, R., \& Canino, G.J. (2007). Assessing diversity among Latinos: Results from the NLAAS. Hispanic Journal of Behavioral Sciences, 29, 510-534.

Gutierrez, P.M. (2006). Integratively assessing risk and protective factors for adolescent suicide. Suicide and Life-Threatening Behavior, 36, 129-135.

Hass, A.P., Hendin, H., \& Mann, J.J. (2003). Suicide in college students. American Behavioral Scientist, 46, 1224-1240.

Heinrich, L.M., \& Gullone, E. (2006). The clinical significance of loneliness: A literature review. Clinical Psychology Review, 26, 695-718.

Hirsch, J.K., Wolford, K., LaLonde, S.M., Brunk, L., \& Morris, A.P. (2007). Dispositional optimism as a moderator of the relationship between negative life events and suicide ideation and attempts. Cognitive Therapy and Research, 31, 533-546.

Joiner, Jr., T.E., \& Rudd, M.D. (2000). Intensity and duration of suicidal crises vary as a function of previous suicide attempts and negative life events. Journal of Consulting and Clinical Psychology, 68, 909-916.

Lewinsohn, P.M., Rohde, P., \& Seeley, J.R. (1996). Adolescent suicidal ideation and attempts: Prevalence, risk factors, and clinical implications. Clinical Psychology: Science and Practice, 3, 25-46.

Olvera, R.L. (2001). Suicidal ideation in Hispanic and mixed-ancestry adolescents. Suicide and Life-Threatening Behavior, 31, 416-427.

Osman, A., Bagge, C., Gutierrez, P.M., Konick, L.C., Kopper, B.A., \& Barrios, F.X. (2001). The Suicidal Behaviors Questionnaire-Revised (SBQ-R): Validation with clinical and nonclinical samples. Assessment, 8, 443-454.

Pantin, H., Coatsworth, J.D., Feaster, D.J., Newman, F.L., Briones, E., Prado, G., et al. (2003). Familias unidas: The efficacy of an intervention to promote parental investment in Hispanic immigrant families. Prevention Science, 4, 189-201.

Peña, J.B., Wyman, P.A., Brown, C.H., Matthieu, M.M., Olivares, T.E., Hartel, D., et al. (2008). Immigration generation status and its association with suicide attempts, substance use, and depressive symptoms among Latino adolescents in the USA. Prevention Science, 9, 299-310.

Queralt, M. (1993). Risk factors associated with completed suicide in Latino adolescents. Adolescence, 28, 831-850.

Rew, L., Thomas, N., Horner, S.D., Resnick, M.D., \& Beuhring, T. (2001). Correlates of recent suicide attempts in a triethnic group of adolescents. Journal of Nursing Scholarship, 33, 361-367.

Russell, D. (1996). UCLA Loneliness Scale (Version 3): Reliability, validity, and factor structure. Journal of Personality Assessment, 66, 20-40.

Russell, D., Peplau, L.A., \& Cutrona C.E. (1980). The revised UCLA Loneliness Scale: Concurrent and discriminant validity evidence. Journal of Personality and Social Psychology, 39, 472-480.

Stravynski, A., \& Boyer, R. (2001). Loneliness in relation to suicide ideation and parasuicide: A population-wide study. Suicide and Life-Threatening Behavior, 31, $32-40$.

Tomoda, A. (1997). Correlations between ratings of experienced and imagined life events by first-year university students in Japan. Psychological Reports, 81, 187-193. 
Triandis, H.C., Bontempo, R., Villareal, M.J., Asai, M., \& Luccca, N. (1988). Individualism and collectivism: Cross-cultural perspectives on self-ingroup relationships. Journal of Personality and Social Psychology, 54, 323-338.

U.S. Census Bureau. (2006). Nation's population one-third minority. Washington, DC: U.S. Government Printing Office.

U.S. Census Bureau. (2008, August 14). An older and more diverse nation by midcentury. Washington, DC: U.S. Census Bureau News. Retrieved November 15, 2009, from http:/ www.census.gov/Press-Release/www/releases/archives/population/012496.html

Wadsworth, T., \& Kubrin, C.E. (2007). Hispanic suicide in U.S. metropolitan areas: Examining the effects of immigration, assimilation, affluence, and disadvantage. American Journal of Sociology, 112, 1848-1885.

Westefeld, J.S., Button, C., Haley, Jr., J.T., Kettmann, J.J., MacConnell, J., Sandil, R., et al. (2006). College student suicide: A call to action. Death Studies, 30, 931-956.

Westefeld, J.S., \& Furr, S.R. (1987). Suicide and depression among college students. Professional Psychology: Research and Practice, 18, 119-123.

Yang, B., \& Clum, G.A. (1994). Life stress, social support, and problem-solving skills predictive of depressive symptoms, hopelessness, and suicide ideation in an Asian student population: A test of a model. Suicide and Life-Threatening Behavior, 24, 127-139. 